\title{
Survey of Schmallenberg virus (SBV) infection in German goat flocks
}

\author{
C. HELMER*, R. EIBACH, P. C. TEGTMEYER, E. HUMANN-ZIEHANK \\ AND M. GANTER \\ Clinic for Swine and Small Ruminants, University of Veterinary Medicine Hannover, Foundation, Germany
}

Received 17 October 2012; Final revision 4 January 2013; Accepted 26 January 2013;

first published online 18 March 2013

\section{SUMMARY}

Animal losses due to abortion and malformed offspring during the lambing period 2011/2012 amounted to $50 \%$ in ruminants in Europe. A new arthropod-borne virus, called Schmallenberg virus (SBV), was identified as the cause of these losses. Blood samples were obtained from 40 goat flocks and tested for antibodies against SBV by ELISA, with $95 \%$ being seropositive. The calculated intra-herd seroprevalence (median 36.7\%, min-max 0-93.3\%) was smaller than in cattle or sheep flocks. Only $25 \%$ of the farmers reported malformations in kids. Statistical analysis revealed a significantly lower risk of goats housed indoors all year-round to be infected by SBV than for goats kept outside day and night. The low intra-herd seroprevalence demonstrates that German goat flocks are still at risk of SBV infection. Therefore, they must be protected during the next lambing seasons by rescheduling the mating period, implementing indoor housing, and continuous treatment with repellents or vaccination.

Key words: Bunyaviruses, estimating, infectious disease, prevalence of disease.

\section{INTRODUCTION}

In summer and autumn 2011 an unknown disease including the clinical signs of diarrhoea, fever and a reduction in milk yield for a short period of time was worrying dairy cattle farmers and veterinarians in The Netherlands and the north-western parts of Germany. In November 2011 the Friedrich-LoefflerInstitute (Federal Research Institute for Animal Health, Germany) succeeded in clarifying these unknown occurrences. Blood samples from affected dairy cattle from a farm near the city of Schmallenberg (North-Rhine Westphalia, Germany)

\footnotetext{
* Author for correspondence: Miss C. Helmer, Clinic for Swine and Small Ruminants, University of Veterinary Medicine Hannover, Foundation, Bischofsholer Damm 15, D-30173 Hannover, Germany.

(Email: Carina.Helmer@tiho-hannover.de)
}

were analysed using a meta-genomic approach with next-generation sequencing, identifying a new virus, provisionally named Schmallenberg virus (SBV) [1].

Between December 2011 and May 2012 a large number of malformations occurred in newborn goat kids and sheep lambs in The Netherlands and the north-western parts of Germany with the highest number of malformations reported in January 2012. Typical clinical signs were arthrogryposis, hydranencephaly, scoliosis and brachygnathia inferior [2, 3]. Most of these deformed lambs were born dead, died soon after birth or had to be euthanized shortly after birth. In many cases only one in a multiple birth was affected while others in the same litter were viable and did not show any malformations.

The large number of deformed lambs and kids warranted strict surveillance of the lambing period. Delivery assistance, fetotomy and caesarean sections 
were indicated more often [4]. In some cases animals had to be euthanized because of a uterine rupture. In addition, the duration of delivery was increased which sometimes led to retentio secundarium followed by endometritis. Moreover, high numbers of embryonic deaths, abortions and stillbirths were described [3].

Phylogenetic analytical investigations showed that SBV belongs to the Simbu serogroup within the genus Orthobunyavirus within the family Bunyaviridae [1]. Shamonda virus, Aino virus and Akabane virus are also members of this group and are all known particularly to infect ruminants. Full-genome and serological investigations by Goller et al. [5] indicated that SBV belongs to the species Sathuperi virus and is a possible ancestor of the reassortant Shamonda virus. Orthobunyaviruses are spread by arthropods (arthropod-borne viruses) and occur in Africa, Asia and Australia. The main vectors of these viruses are biting midges, primarily Culicoides spp. and mosquitoes [1]. Two real-time reverse transcriptasepolymerase chain reaction (rRT-PCR) studies on the presence of SBV RNA in Culicoides spp., performed in autumn 2011 in Belgium and Denmark, revealed that several Culicoides spp. (C. obsoletus complex, C. dewulfi, $C$. chiopterus) had tested positive for SBV, strongly indicating that these species are relevant natural vectors for the virus [6, 7]. Direct animal-to-animal transmission was considered to be unlikely. Vertical transmission from the ewe/doe to the fetus during gestation is possible and can cause malformations. Based on the pathogenesis of Akabane virus it can be assumed that an infection from day 1 until day 28 after mating leads to embryonic death, delivery of small lambs, decreased fertility and stillbirth. If the virus infects the ewe/doe during day 28 to day 56 of pregnancy deformed lambs with arthrogryposis, hydranencephaly, scoliosis, torticollis and brachygnathia inferior can be seen besides mummification, abortion and stillbirth. After day 56 of gestation the fetus is expected to fight the virus with its matured immune system although mummification, abortion and stillbirth can still be observed in some cases [8]. It can be assumed that an infection of the ewe/doe in the second month of gestation is most dangerous for fetuses as it can cause malformations. Whether the accepted pathogenesis of Akabane virus can be completely transferred to SBV still remains to be confirmed.

Arthrogryposis is characterized by stiffening of the joints and malpositions of the extremities. The clinical signs can be explained by an abnormal fetal development of the motor neurons of the ventral horn of the spinal cord. These disorders cause an inadequate development of the muscular system with subsidiary neurogenic muscle atrophy followed by ankylosis of the joints [9]. Many of the deformed lambs show neurological failures such as a decreased sucking reflex, blindness and movement disorders, with some lambs being unable to stand or walk. Pathomorphological findings in these lambs revealed different stages of cerebral malformations ranging from hydrocephalus internus, hypoplasia of the cerebellum to anencephaly, porencephaly and hypoplasia or agenesia of the spinal cord [10].

Farmers retrospectively reported cases of languor and diarrhoea in some adult sheep and goats during the period when SBV infection of the flock might have taken place. SBV infection in early gestation can cause embryonic death and resorption, resulting in a return to oestrus although the animals have been mated. Fertility in the next oestrus seems to be reduced. If the ram has been removed early from the flock, these does/ewes remain barren.

A SBV infection can be verified either by detection of viral RNA by rRT-PCR or virus culture or by serological techniques such as the serum neutralization test, indirect immunofluorescence test or indirect ELISA test [4]. The detection of viral antigen in adult animals is possible during the febrile period of the infection by EDTA blood sampling. The detection of viral antigen in neonates with congenital malformations is considered to be most pronounced in brain tissue (a pooled sample of cerebrum, cerebellum and brainstem), the spleen, blood and meconium [9]. However, only $20-80 \%$ of the brain tissue samples tested positive by RT-PCR although there were many indications that a SBV infection existed in the flock [4].

In March 2012 the SBV infection was given the status of a notifiable animal disease in Germany and The Netherlands. In Germany 48 goat flocks, 1048 cattle herds and 880 sheep flocks were notified to be positive for SBV [11] with a high estimate of unreported cases. Meanwhile, several countries bordering Germany and The Netherlands (Austria, Belgium, France, Luxembourg, Denmark, Switzerland, Poland) as well as Italy, Spain, Great Britain, Ireland, Northern Ireland, Sweden, Norway and Finland [11] also had proven cases of SBV infection.

The reason for this serosurvey was to acquire the scale of the distribution of SBV infection as a newly emerging disease throughout goat flocks in the most 
SBV-affected federal states of Germany. A SBVspecific questionnaire was developed to collect data on SBV infections in order to obtain an overview of clinical symptoms that might be caused by the virus, possible paths of its distribution and possibilities of protecting seronegative animals against a SBV infection during the hazardous second month of gestation.

\section{METHODS}

Examinations were performed in 40 randomly selected goat flocks in the six federal states of Germany which were thus far the most SBV-affected federal states of Germany (11 flocks in North Rhine-Westphalia, ten flocks in Lower Saxony, seven flocks in Hesse, seven flocks in Schleswig-Holstein, four flocks in SaxonyAnhalt and one flock in Mecklenburg-Western Pomerania) (Fig. 1). Serum samples were gained from about 1065 adult female goats ( $>1$ year) in order to detect the antibody status of SBV of these flocks.

According to the Federal Statistical Office of Germany a total of 149936 goats housed on 11219 farms were kept in Germany in March 2010 [12]. Of these, 75544 goats are kept for breeding (about $50 \%$ ). The average flock size is 13.4 goats per farm. About 10000 goat farms in Germany only keep 1-19 animals per flock [12]. The number of goats that need to be tested to detect the presence of antibodies to SBV at an expected seroprevalence of $20 \%$ with a $95 \%$ level of confidence is 14 . Of the remaining 1272 goat flocks large enough to be surveyed for the study 288 flocks were located in the six federal states tested for this serosurvey. Therefore 40 goat flocks were tested for antibodies against SBV by ELISA $(13 \cdot 8 \%)$.

Concurrently, a SBV-specific questionnaire was conducted in the above-mentioned flocks by veterinarians of the Clinic for Swine and Small Ruminants in order to collect data on the epidemiology and the distribution of the virus within German goat flocks.

\section{Ethical statement}

The experimental procedures on the animals were performed in accordance with the principles outlined by the European Convention for the Protection of Vertebrate Animals used for Experimental and other Scientific Purposes. The blood samples were taken by veterinarians of the University of Veterinary Medicine Hannover, Foundation.

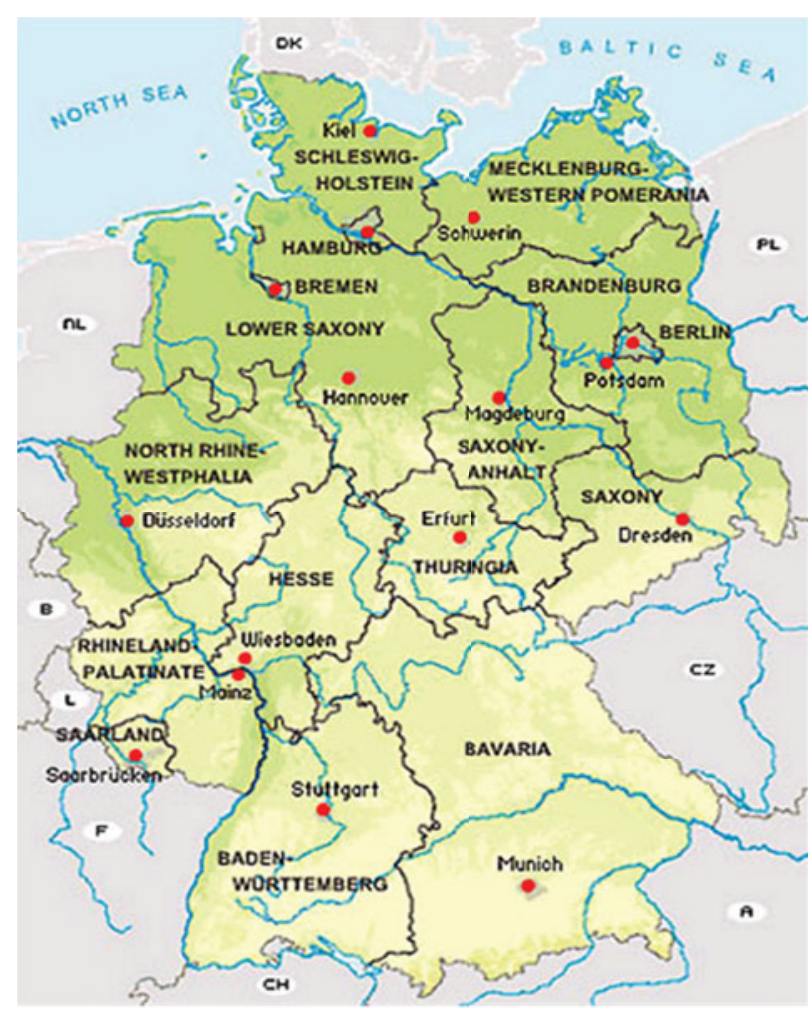

Fig. 1 [colour online]. Map of Germany showing the federal states (source: http://www.nationsonline.org/maps/ german_states_map.jpg). Accessed 2 October 2012.

\section{Samples}

Between January and the beginning of June 2012, 1065 serum samples were obtained from adult female goats ( $>1$ year). A minimum of 14 (12 flocks) and a maximum of 30 ( 22 flocks) serum samples were gained from the individual flocks depending on the flock size. In six cases the entire flock was tested (three flocks with 20 samples, two flocks with 50 samples and one flock with 77 samples, respectively). Sampling was performed by venepuncture (S-Monovette ${ }^{\circledR}$, Sarstedt, Germany) of the vena jugularis externa or of the vena cava cranialis [13]. The blood was centrifuged (at $10000 \mathrm{~g}$ ) for $10 \mathrm{~min}$ and the supernatant (serum) was stored at $4{ }^{\circ} \mathrm{C}$ until analysis. Fourteen serum samples of each goat flock were sent to the FriedrichLoeffler-Institute to be tested for SBV antibodies by ELISA. The remaining samples of flocks large enough to take a random sample of $\geqslant 15$ serum samples were tested for antibodies with ELISA by laboratory personnel of the Clinic for Swine and Small Ruminants of the University of Veterinary Medicine Hannover, Foundation. The intra-herd prevalence of each individual flock was calculated by taking both results into account. 


\section{ELISA test}

At both institutes serum samples were analysed for the presence of antibodies against SBV utilizing a commercial ELISA (ID Screen ${ }^{\circledR}$, Schmallenberg virus Indirect, IDvet Laboratories, France), according to the manufacturer's instructions. The test can be used with individual bovine, ovine and caprine serum or plasma. If anti-SBV antibodies are present, they form an antibody-antigen complex and can be detected by a colour change of the added substrate.

Following the manufacturer's recommendations, samples presenting a $\mathrm{S} / \mathrm{P}$ (sample/positive) percentage $\leqslant 60 \%$ are considered negative, those $>60 \%$ and $\leqslant 70 \%$ are considered doubtful and those $>70 \%$ are considered positive by the ELISA test used.

\section{SBV-specific questionnaire}

The initial version of the questionnaire was created and established in cooperation with the Institute for Epidemiology of the Friedrich-Loeffler-Institute (Institute for Epidemiology, Wusterhausen, Germany), mainly dealing with SBV infections in cattle. This initial questionnaire was shortened and adapted to SBV infections and their possible reasons in small ruminants by the authors (see online Supplementary material). The primary function of this questionnaire is to collect additional and standardized data from the farms on housing conditions and handling of the animals, clinical signs observed in the flocks and treatment of the stock with focus on the usage of repellents.

Housing conditions and treatment with repellents are very important factors for the study, particularly with regard to the exposure of the animals to biting midges which is a precondition for the outbreak of an SBV infection. Another very important factor is the time of mating since only an infection during gestation is currently presumed to cause malformations in neonates.

The investigation of the clinical signs indicated whether a particular farm was affected by an SBV outbreak or not. The reason for this investigation was to understand the range of symptoms which might be caused by a SBV infection.

\section{Statistical analysis}

The comparisons of potential significant correlations between federal state (except of MecklenburgWestern Pomerania, where only one farm was tested), clinical signs, housing conditions, treatment with repellents, exposure to wetland/woodland, time of mating and the percentage of intra-herd prevalence was statistically analysed using SAS v. 9.1 (SAS Institute Inc., USA). Statistical significance was tested by Kruskal-Wallis and Wilcoxon two-sample tests. A significant correlation was confirmed if $P<0 \cdot 05$.

\section{RESULTS}

\section{Clinical signs}

Only ten of the 40 goat flocks tested showed clinical signs of a SBV infection (i.e. arthrogryposis, hydranencephaly, scoliosis, brachygnathia, stillbirth, abortion) according to reports by the farmers. Three of these farms were severely affected with economic losses up to $50 \%$ due to loss of kids and decreased milk production. The most affected farm was located in the north-eastern part of the Harz Mountains (Saxony-Anhalt, Germany) and had a flock of 62 dairy goats (status of May 2012). It also had 24 beef cattle and eight sheep, the beef cattle, sheep and dairy goats were all housed in different stables and on different pastures and therefore did not come into contact with each other. In summer 2011 the farmer observed mild diarrhoea and a reduction in milk yield of up to $50 \%$ in the goat flock while the other animals seemed to be unaffected. All animals were treated twice with repellents (cyfluthrin, Bayofly pour-on ${ }^{\circledR}$, Bayer, Germany) in April 2011 and in August 2011. The farm area is surrounded by wetlands, moor and woodland. During delivery (beginning of February until the end of April) the farmer noticed an abnormally high number of abortions in the goat flock in the early stage of gestation as well as in the late stage of gestation. Eleven of the goats were barren although the owners had observed the mating of these animals. Twelve does were not able to produce any milk after delivery so that the milk yield continued to fall. Twenty kids were born with congenital malformations and were either born dead or had to be euthanized. In most cases only one in a multiple birth was affected while others in the same litter were viable. The malformations were reflected in stiffening of the joints, malposition of the extremities and scoliosis leading to obstructed labour. The kids also showed neurological failures ranging from reduced sucking reflex, ataxic movements to the inability to stand or walk. The other two severely affected farms (located in 


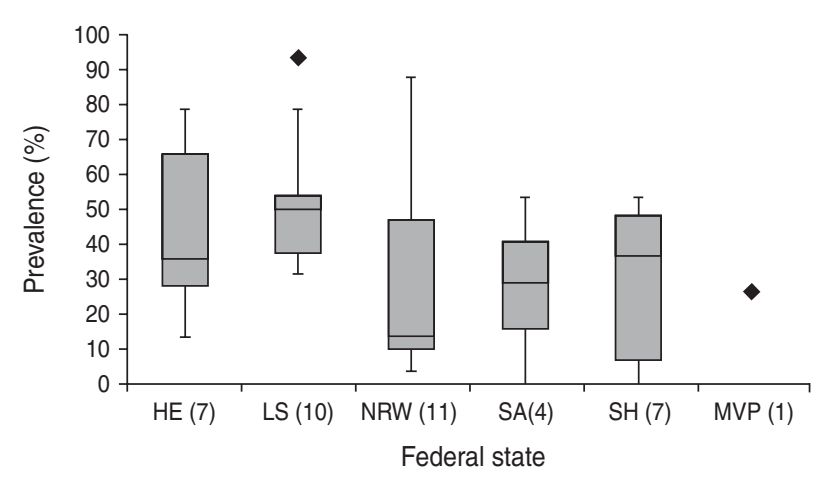

Fig. 2. Seroprevalence of Schmallenberg virus (SBV) in different German federal states. HE, Hesse; LS, Lower Saxony; NRW, North Rhine-Westphalia; SA, SaxonyAnhalt; SH, Schleswig-Holstein; MVP, MecklenburgWestern Pomerania. $P$ values: none of the calculated $P$ values were significant; MVP was not taken into consideration because there was only one farm tested.

Lower Saxony and Hesse) are also surrounded by wetland and woodland. Both of the farmers treated their flocks with repellents (deltamethrin, Butox 7.5 pour-on ${ }^{\mathbb{R}}$, Intervet Deutschland GmbH, Germany). One of the flocks was kept indoors all year-round whereas the other flock was constantly out on pasture. Neither farmer observed any clinical signs in the adult goats but experienced large losses due to newborn kids with malformations and neurological failures.

The farmers of the other seven goat flocks who reported clinical signs of SBV only noticed isolated cases of abortion, stillbirth or congenital malformations in newborn kids and did not notice any clinical signs in the adult goats.

\section{Serological response}

Antibodies against SBV were detected by ELISA in $38 / 40(95 \%)$ goat flocks tested with an intra-herd prevalence ranging from $3 \cdot 3 \%$ to $93 \cdot 3 \%$, while most of the flocks that tested positive for a SBV infection did not show any clinical signs at all. The ten flocks showing clinical signs of a SBV infection had an intra-herd prevalence ranging from $10 \%$ to $53 \cdot 3 \%$. The calculated median in German goat flocks is $36.7 \%$. The intra-herd prevalence differs from one federal state to another. The calculated median for North Rhine-Westphalia was $13 \cdot 3 \%$ which is the lowest value whereas the calculated median for Lower Saxony was $50 \%$ which is the highest value for intraherd prevalence throughout German goat flocks (Fig. 2).
The goat flock located in the Harz region (SaxonyAnhalt, Germany) which was clinically the most affected had an intra-herd prevalence of $53 \cdot 3 \%$. The other two severely affected farms had an intra-herd prevalence of $35 \%$.

\section{Evaluation of the SBV-specific questionnaire}

The evaluation of the questionnaires and the statistical analysis was performed by the first author (C.H.).

As previously mentioned only ten $(25 \%)$ farms out of the 40 interviewed reported an outbreak of SBV infection, while only seven farms reported isolated cases of malformed neonates. With regard to the treatment with repellents the questionnaire indicated the following results: Not more than $11(27 \cdot 5 \%)$ out of 40 farmers treated their goats with repellents while $28(70 \%)$ of the 40 farmers did not treat their animals with repellents at all. One farm started treatment in March 2012, so they were unable to provide any information about the usage of repellents on the farm of origin in 2011. The active agents applied were pyrethroids as a pour-on insecticide [nine times: deltamethrin (seven times), cyfluthrin (twice)] and macrocyclic lactones subcutaneously or as a pour-on insecticide (twice: ivermectin subcutaneously, eprinomectin as a pour-on insecticide; each of them once).

Moreover, the questionnaire revealed that most of the ten farms affected by a clinical SBV infection are surrounded either by wetland or by woodland. Two of the most affected farms are even surrounded by both.

Furthermore, it does not go unnoticed that almost all flocks with a low intra-herd prevalence are kept indoors during the whole year or at least housed in barns at night $(35 \%)$.

In addition, the evaluation of the survey revealed that flocks with a high intra-herd prevalence, not showing any clinical signs, had a late mating period, approximately from October 2011 until January 2012.

\section{Statistical analysis}

The results of the Kruskal-Wallis and Wilcoxon twosample tests revealed that the only significant correlation existed between housing conditions (indoors $v s$. outdoors) and percentage of intra-herd prevalence ( $P=0 \cdot 0001$, Fig. 3). A significant correlation between the other five comparisons with the intra-herd prevalence (federal state, clinical sings, treatment with 


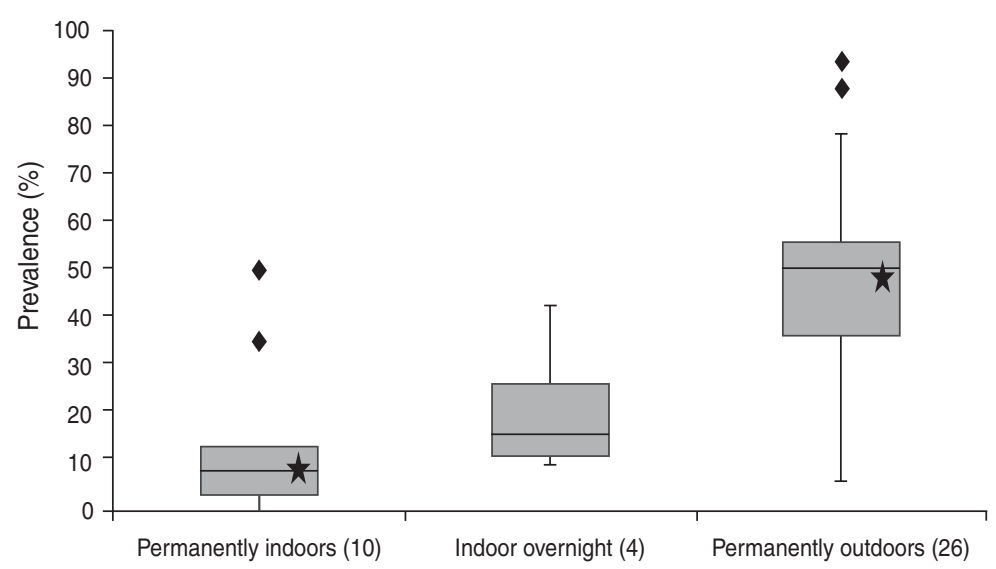

Fig. 3. Comparison of prevalence and housing conditions. The calculated $P$ values for permanently indoors $v s$. permanently outdoors (marked with a star) is 0.0001 ; this $P$ value is significant $(<0.05)$; the subitem 'indoors overnight' was not taken into consideration for statistical analysis because only four flocks were kept under these conditions.

repellents, exposure to wetland/woodland, time of mating) could not be confirmed by the statistical tests.

\section{DISCUSSION}

This serosurvey outlines the wide range of intra-herd prevalence for SBV within German goat flocks.

Serological analyses showed that only two of the 40 examined flocks tested negative for SBV. The other 38 flocks had an intra-herd prevalence ranging from $3.3 \%$ to $93 \cdot 3 \%$. The calculated median in German goat flocks is $36.7 \%$ which is far smaller than the median of the intra-herd prevalence in cattle (about 80\% throughout German cattle herds [14] and about 72.5\% in dairy cows in The Netherlands [15]), and sheep (60\% in Lower Saxony, investigations of the Clinic for Swine and Small Ruminants, University of Veterinary Medicine Hannover, Foundation, Germany, unpublished observations). Interestingly the intra-herd prevalence differs from one federal state to another (Fig. 2). In particular, the low intra-herd prevalence in North Rhine-Westphalian goat flocks is surprising, due to the fact that the disease occurred there very early [first detection of SBV in Schmallenberg (North RhineWestphalia) in November 2011] [1].

Although $95 \%$ of the flocks tested positive by ELISA, only ten flocks reported clinical signs of a SBV infection with only three flocks having a positive RT-PCR result of deformed kids which were sent to a veterinary investigation laboratory.

One possible reason for these findings might be the different mating periods on the farms. Many of the farms with a high seroprevalence did not suffer from any clinical signs and mated their does in the colder months of the year $(40 \%$ from October until January). It can be assumed that many of these flocks were infected during the warm period of the year but due to the fact that the animals were not pregnant at that time no clinical signs could be observed in the following lambing period. A mild infestation can be assumed in these cases.

As the questionnaire shows, the two flocks that tested negative for SBV were flocks which housed their does indoors all year-long so that exposure to biting midges was likely to be low. The low intra-herd prevalence in North Rhine-Westphalia (Fig. 2) can also be explained by keeping animals indoors. Four out of eleven farms tested in North Rhine-Westphalia kept their goats indoors all yearlong and on two farms flocks were housed in barns overnight (Table 1). Statistical tests confirmed that there is a significant correlation between housing conditions (indoors $v s$. outdoors) and percentage of the intra-herd prevalence of a flock. Figure 3 illustrates this correlation by showing that the percentage of the intra-herd prevalence rises steadily from permanently indoors via indoors overnight to permanently outdoors. The subitem 'indoors overnight' was not taken into consideration for statistical analysis because there were only four flocks housed under these conditions (Table 1).

Furthermore, the questionnaire reveals that the ten flocks affected by a clinical SBV infection are almost all located in areas with wetland or woodland. The flock which was the most affected is located in the north-eastern part of the Harz mountains (SaxonyAnhalt, Germany), surrounded by moor and woodland, which explains the high risk of infection in this 
Table 1. Outcomes of the SBV-specific questionnaire

\begin{tabular}{|c|c|c|c|c|c|c|c|c|c|}
\hline Farm & Type of use & $\begin{array}{l}\text { Federal } \\
\text { state }\end{array}$ & $\begin{array}{l}\text { Clinical } \\
\text { signs }\end{array}$ & $\begin{array}{l}\text { PCR } \\
\text { results }\end{array}$ & $\begin{array}{l}\text { Permanent } \\
\text { housing }\end{array}$ & Treatment with repellents & $\begin{array}{l}\text { Exposure to } \\
\text { woodland/wetland }\end{array}$ & Mating period & Prevalence \\
\hline 1 & Milk & SA & No & - & Yes & No information & No & Feb.-Mar. & $0 \cdot 00 \%$ \\
\hline 2 & Meat & $\mathrm{SH}$ & No & - & Yes & Yes (deltamethrin, once) & No & Nov.-Dec. & $0 \cdot 00 \%$ \\
\hline 3 & Milk & NRW & No & - & Yes & No & No & No information & $3 \cdot 30 \%$ \\
\hline 4 & Milk & NRW & No & - & Yes & No & Yes & Aug.-Dec. & $3 \cdot 30 \%$ \\
\hline 5 & Milk & $\mathrm{SH}$ & No & - & No & No & No & Sep.-Oct. & $6 \cdot 70 \%$ \\
\hline 6 & Milk & $\mathrm{SH}$ & No & - & Yes & No & No & Sep.-Nov. & $6 \cdot 70 \%$ \\
\hline 7 & Milk & NRW & Yes & Positive & Indoors overnight & No & No & Aug.-Dec. & $10 \cdot 00 \%$ \\
\hline 8 & Milk & NRW & No & - & Yes & No & Yes & July-Feb. & $10 \cdot 00 \%$ \\
\hline 9 & Milk & NRW & No & - & Indoors overnight & No & Yes & Aug.-Dec. & $11 \cdot 60 \%$ \\
\hline 10 & Milk & $\mathrm{HE}$ & Yes & Positive & Yes & Yes (deltamethrin, once) & Yes & Aug.-Jan. & $13 \cdot 30 \%$ \\
\hline 11 & Milk & NRW & Yes & - & Yes & No & No & Aug.-Jan. & $13 \cdot 30 \%$ \\
\hline 12 & Hobby breeder & SA & No & - & Indoors overnight & No & No & Oct. & $20 \cdot 60 \%$ \\
\hline 13 & Milk & MVP & Yes & - & No & Yes (deltamethrin, twice) & Yes & Sep.-Nov. & $26 \cdot 30 \%$ \\
\hline 14 & Milk & $\mathrm{HE}$ & No & - & No & No & Yes & Aug.-Feb. & $26 \cdot 90 \%$ \\
\hline 15 & Hobby breeder & $\mathrm{HE}$ & No & - & No & Yes (eprinomectin, once) & Yes & Oct.-Dec. & $28 \cdot 60 \%$ \\
\hline 16 & Animal park & LS & Yes & Negative & No & No & Yes & Nov.-Jan. & $31 \cdot 40 \%$ \\
\hline 17 & Milk & LS & Yes (severely) & - & Yes & Yes (deltamethrin, twice) & No & Aug.-Jan. & $35 \cdot 20 \%$ \\
\hline 18 & Milk & HE & Yes (severely) & - & No & Yes (deltamethrin, once) & Yes & Aug.-Dec. & $35 \cdot 70 \%$ \\
\hline 19 & Milk & LS & No & - & No & Yes (deltamethrin, once) & Yes & Aug.-Jan. & $36 \cdot 70 \%$ \\
\hline 20 & Milk & SH & No & - & No & Yes (cyfluthrin, once) & Yes & Oct. & $36 \cdot 70 \%$ \\
\hline 21 & Meat & SA & No & - & No & No & No & Sep.-Oct. & $36 \cdot 80 \%$ \\
\hline 22 & Milk & $\mathrm{LS}$ & Yes & Negative & No & No & Yes & Aug.-Dec. & $40 \cdot 30 \%$ \\
\hline 23 & Hobby breeder & NRW & No & - & Indoors overnight & No & No & Aug. & $42 \cdot 90 \%$ \\
\hline 24 & Milk & NRW & No & - & No & No & Yes & Aug.--Nov. & $43 \cdot 30 \%$ \\
\hline 25 & Milk & $\mathrm{SH}$ & Yes & - & No & No & Yes & Aug.-Sep. & $46 \cdot 70 \%$ \\
\hline 26 & Meat & LS & No & - & No & No & No & Oct.-open end & $50 \cdot 00 \%$ \\
\hline 27 & Milk & NRW & No & - & No & No & Yes & Aug. & $50 \cdot 00 \%$ \\
\hline 28 & Research & LS & No & - & Yes & No & No & Aug.-Jan. & $50 \cdot 00 \%$ \\
\hline 29 & Milk & SH & No & - & No & No & No & No information & $50 \cdot 00 \%$ \\
\hline 30 & Hobby breeder & LS & No & - & No & No & Yes & June-Aug. & $51 \cdot 20 \%$ \\
\hline 31 & Meat & SH & No & - & No & No & Yes & Dec.-Feb. & $53 \cdot 30 \%$ \\
\hline 32 & Milk & SA & Yes (severely) & Positive & No & Yes (cyfluthrin, twice) & Yes & Oct.-Dec. & $53 \cdot 30 \%$ \\
\hline 33 & Milk & LS & No & - & No & Yes (deltamethrin, twice) & Yes & Oct.-Nov. & $54 \cdot 80 \%$ \\
\hline 34 & Meat & NRW & No & - & No & No & Yes & Year-round & $56 \cdot 70 \%$ \\
\hline 35 & Hobby breeder & $\mathrm{HE}$ & No & - & No & No & No & Aug.-Sep & $64 \cdot 30 \%$ \\
\hline 36 & Meat & $\mathrm{HE}$ & No & - & No & No & Yes & June-Sep & $66 \cdot 70 \%$ \\
\hline 37 & Hobby breeder & LS & No & - & No & No & Yes & Year-round & $78 \cdot 60 \%$ \\
\hline 38 & Hobby breeder & HE & No & - & No & No & No & May-Oct & $78 \cdot 60 \%$ \\
\hline 39 & Hobby breeder & NRW & No & - & No & Yes (ivermectin, once) & No & Oct & $87 \cdot 50 \%$ \\
\hline 40 & Milk & LS & No & - & No & No & No & Sep.-Oct & $93 \cdot 30 \%$ \\
\hline
\end{tabular}

HE, Hesse; LS, Lower Saxony; NRW, North Rhine-Westphalia; SA, Saxony-Anhalt; SH, Schleswig-Holstein; MVP, Mecklenburg-Western Pomerania. 
area because of the high exposure to biting midges, primarily Culicoides spp. and mosquitoes. Additionally, the goats on this farm are on pasture both day and night. In general, developmental sites of Culicoides spp. are poorly known. Larval stages of Culicoides spp. are dependent on humid substrate and develop aquatically. They occur in wet areas, especially close to swamps and moor landscapes, liquid manure, in wet spots, trenches or other stagnant waters and even in sandy subsoil close to the sea. Females of many Culicoides spp. feed on nutrient-rich blood of mammals or birds in order to introduce oogenesis and are therefore potential vectors of pathogenic agents. Most of the Culicoides spp. are crepuscular, having peaks at sunset and sunrise, or nocturnal [16]. As a monitoring programme of Culicoides spp. at 20 locations in north-west Germany revealed, Culicoides spp. show a seasonal distribution with the highest numbers of individuals in spring and summer [17]. During the winter months the population density decreases but even in the coldest months of January and February a very small population density of Culicoides spp. is present. The diversity of Culicoides spp. was highest during the summer months. As C. obsoletus remains the only species present in winter and dominates in summer, it seems to be the most resistant [17]. Due to these facts C. obsoletus might be a year-round active vector for SBV as already assumed for bluetongue virus. Several studies on seasonal dynamics of biting midges outline that $C$. obsoletus spp. are most abundant during May-June and AugustSeptember with a total range from March to November [17-19]. Mehlhorn et al. discovered that C. obsoletus, in particular, is found throughout the year and also inside or close to stables during the winter months in North Rhine-Westphalia (Germany) [20].

The last cases of congenital malformations in newborn goat kids were reported at the beginning of May 2012 at an animal park in Lower Saxony, Germany. It might be important that this flock with late infections of SBV is a family park with a wide range of different animal species (horses, donkeys, cattle, pigs, sheep, goats, camelids, birds, reptiles) located around a former gravel quarry with a lake in the centre of the park. The goats are housed in an open shed and have contact with other ruminants. As described above the period of contracting congenital malformations extends from day 28 until day 56 of gestation [8]. With a gestation length of $\sim 150$ days the infection must have occurred around January 2012. During this time of year the Culicoides population is expected to be very low. As mentioned earlier, C. obsoletus are present even in the colder winter months $[17,20]$. Hence, a possible SBV infection during the cold months should be taken into consideration.

A surprising result of our serosurvey was that in the majority of cases only one in a multiple birth was suffering from congenital malformations caused by a SBV infection. Others in the same litter were viable and did not show any clinical signs whereas both male and female lambs were affected. This might be explained by placentation in small ruminants. The gestation period of small ruminants is $148-153$ days. Twenty-seven percent of goats bear singletons, $57 \%$ bear twins and $15 \cdot 6 \%$ even bear triplets or quadruplets [21]. Placentation begins on day 31 with the fusion of allantois and chorion. In the fetuses a fusion between the chorions and the allantoises can be observed in most cases. Even the amnia can join up. In small ruminants, as opposed to cattle, a vascular anastomosis between fetuses is an exception [22]. These facts might explain why in numerous cases of malformations only one in a multiple birth was affected. Based on the pathogenesis of Akabane virus it is believed that SBV is transmitted vertically [3, 8]. This means that SBV has the ability to pass through the placenta and infect the fetus. Due to the non-existent fetal vascular anastomoses the virus cannot be transmitted from one fetus to another. Therefore, it might be possible that in a multiple birth only one of the litter suffers from malformation while the others remains unaffected. The large number of differences between multiple births is marked.

The evaluation of the SBV-specific questionnaire regarding the treatment of the animals with repellents showed that the period of treatment with repellents in the different flocks differed greatly and extended from April to December 2011. Only four farmers treated their flock twice. Contrary to expectations two of these farms were affected the most by clinical signs of a SBV infection. This can be explained by the fact that none of the farmers treated their flock during the mating period so that there was no protection of the does against biting midges during the hazardous second month of gestation.

A pilot study on deltamethrin treatment of cattle and sheep against biting midges showed that animals treated with deltamethrin on the neck or along the back are protected against biting midges for up to 4-5 weeks [23].A subsequent study revealed that deltamethrin (Butox $7 \cdot 5$ pour-on ${ }^{\circledR}$ ) remained effective for at least up to 28 days even when the animals were 
exposed to rain twice weekly over a 4-week period [24]. Field-based tests on deltamethrin insecticides against adult Culicoides biting midges showed that the persistence of the lethal effect of Butox 7.5 pour-on is estimated to be $<10$ days [25].

A study of Culicoides spp. associated with sheep in The Netherlands revealed that Culicoides spp. are most active at sunset [26]. Twenty-four $(57 \cdot 5 \%)$ out of the 40 goat flocks tested for antibodies against SBV are dairy goat flocks. Therefore, the goats are either kept indoors all year long or at least are brought into barns before the evening milking, i.e. before sunset. This might also be a reason for the lower seroprevalence in goat flocks because at the main flight time of Culicoides spp. the goats are protected by shelter. An Australian study on protection of cattle against Culicoides spp. by shelter and chemical treatment showed that neither shelter nor chemical treatment alone can currently eliminate the risk of exposure to biting midges but can at least reduce it [27]. Baldet et al. discovered that in France, Belgium and The Netherlands Culicoides spp. entered animal housing quite freely [28] which again suggests that animal housing alone cannot reliably protect animals against biting midges. Most German and Dutch dairy cattle farms house their cows all year-round but nevertheless the estimated seroprevalence for dairy cattle in The Netherland is $72.5 \%$ [15] which is clearly higher than the calculated median $(36.7 \%)$ for goat flocks tested for this survey. However, this study revealed that the risk of a SBV infection is significantly lower for those goats kept indoors all year-round. One possible explanation for these findings is the fact that almost all dairy cattle herds in Germany and The Netherlands are housed on slatted floors with cesspools below. The accumulation and storage of the very liquid and nutritious cow dung might be an adequate breeding site for Culicoides spp. In contrast to cow dung goat droppings are very arid and solid which makes it hard for the larval stages to migrate into the dung. A survey of Culicoides developmental sites in Spain showed that most of the Culicoides midges collected were found in composted manure, followed by the farm corner and in fresh manure whereas no midges were found in pure sheep droppings or soil inside the sheep stable [19]. With goat and sheep droppings and soil being similarly structured these findings might also be transferred to goat droppings and soil inside goat stables. This hypothesis might be a possible explanation for the lower seroprevalence in goats than in cattle housed all year-round and needs to be investigated more precisely in further studies.

Summarizing these findings we are not able to recommend a method which is able to prevent a SBV infection only by treatment with repellents or by shelter. Nevertheless, we advise farmers to treat their goat flocks with pharmaceuticals against external parasites every 2 weeks in the main flight time of biting midges during the summer months (May-September) if mating is scheduled at about that time of year. Does should be protected against biting midges by utilizing repellents at least during the second month of gestation because of the high risk of a fetal infection in this period of gestation. Bucks should be protected against biting midges beginning 6 weeks ahead of the mating period up to the end of mating in order to prevent fever which could result in reproductive failures. Due to the significant correlation between housing conditions and percentage of intra-herd prevalence permanent housing is favourable. If daytime grazing is necessary does should be brought indoors before sunset. This might also be an option to reduce exposure to biting midges. Insect traps might be helpful to decrease the amount of Culicoides spp. in barns.

Another recommendation could be to reschedule the mating period in these months in which the population density of Culicoides spp. is believed to be low, i.e. under German conditions mating should start at the earliest in October, even better in November although it must be acknowledged that $C$. obsoletus might be present in decreased abundance even in the cold winter months.

Summing up all results it becomes obvious that infestation with SBV in German goat flocks is not even roughly similar to the infestation in cattle and sheep flocks, as goat flocks in Germany are still exposed to the risk of a SBV infection resulting in large economic losses. Therefore, the animals must be protected either by rescheduling the mating period to late autumn, keeping the animals in barns year-round or at least before sunset and overnight, and implementing stringent regulations regarding continuous treatment with repellents over the summer months to avoid infections during the most susceptible period of gestation (second month). SBV infections might also be controlled by moving susceptible animals into endemic regions in time to develop immunity before they are first mated. Nevertheless details on resilience and duration of acquired immunity to SBV are still unclear.

Another possibility would be vaccination. However, there is yet no licensed vaccine on the market. 
Different pharmaceutical companies are working on an effective vaccine against SBV which, in all probability, will not be available before 2013 at the earliest. Further progress of the disease remains to be observed in the next lambing periods.

\section{SUPPLEMENTARY MATERIAL}

For supplementary material accompanying this paper visit http://dx.doi.org/10.1017/S0950268813000290.

\section{ACKNOWLEDGEMENTS}

This work was financially supported by the German Federal Ministry of Food, Agriculture and Consumer Protection (BMELV) through the Federal Office for Agriculture and Food (BLE), grant number 2812HS008. We are very grateful for the support of the Friedrich-Loeffler-Institute (Institute for Federal Animal Research, Germany) who provided ELISA results and cooperated in establishing the SBV-specific questionnaire. We also thank the Lower Saxony State Office for Consumer Protection and Food Safety, the CVUA Münsterland-Emscher-Lippe and the State Office for Consumer Protection of Saxony-Anhalt for providing PCR results of the flocks tested. Furthermore, we thank Frances Sherwood-Brock for reading the English manuscript. The authors also thank the laboratory personnel of the Clinic for Swine and Small Ruminants of the University of Veterinary Medicine Hannover, Foundation for their excellent laboratory technical assistance. Finally we thank the farmers from all of the 40 farms investigated for the study for their support.

\section{DECLARATION OF INTEREST}

None.

\section{REFERENCES}

1. Hoffmann B, et al. Orthobunyavirus in cattle. Emerging Infectious Diseases 2012; 18: 469-472.

2. DFNVC - The Video Conference Service of the German Research Network. Malformations in lambs and calves induced by a new Orthobunyavirus. (https://://webconf. vc.dfn.de/p68qfv0gv0u/). Accessed 15 April 2012.

3. van den Brom R, et al. Epizootic of ovine congenital malformations associated with Schmallenberg virus infection. Tijdschrift voor Diergeneeskunde 2012; 137: 106-111.

4. Ganter M, Köß C. Schmallenberg virus -a new virus infection in Central Europe, which is transferred to ruminants by vectors causing malformations. Klauentierpraxis 2012; 20: 57-63.

5. Goller KV, et al. Schmallenberg virus as possible ancestor of Shamonda virus. Emerging Infectious Diseases 2012; 18: 1644-1646.

6. Rasmussen LD, et al. Culicoides as vectors of Schmallenberg virus. Emerging Infectious Diseases 2012; 18: 1204-1206.

7. De Regge N, et al. Detection of Schmallenberg virus in different Culicoides ssp. by real-time RT-PCR. Transboundary and Emerging Diseases 2012; 59: 471-475.

8. The Center for Food Security and Public Health, Iowa state University. Akabane disease (http://www. cfsph.iastate.edu/Factsheets/pdfs/akabane.pdf). Accessed 5 May 2012.

9. Peters M, Brügmann M. Pathomorphology of SBVassociated malformations and SBV diagnostics of the investigative offices. LANUV (North-Rhine Westphalia State Agency for Nature, Environment and Consumer Protection). Conference on Schmallenberg Virus, 6 February 2012, Münster, Germany.

10. Herder V, et al. Salient lesions in domestic ruminants infected with the emerging so-called Schmallenberg virus in Germany. Veterinary Pathology 2012; 49: 588-591.

11. Friedrich-Loeffler-Institute (FLI), Federal Research Institute for Animal Health, Germany. Schmallenberg virus. (http://www.fli.bund.de/de/startseite/aktuelles/tierseuchengeschehen/schmallenberg-virus.html). Accessed 10 December 2012.

12. Federal Statistical Office of Germany. Viehhaltung der Betriebe Agrarstrukturerhebung Fachserie 3 Reihe 2.1·3-2010 (https://www.destatis.de/DE/Publikationen/ Thematisch/LandForstwirtschaft/ViehbestandTierische Erzeugung/Viehhaltung2030213109004.pdf?_blob= publicationFile). Accessed 12 December 2012.

13. Ganter M, Hermann J, Waibl H. Blood sampling from the vena cava cranialis in sheep and goat using sampling systems armed by canula. Tierärztliche Praxis. Ausgabe Großtiere Nutztiere 2001; 29: 37-40.

14. Schirrmeier H. 'Schmallenberg pot-pourri': about a new disease which is no emerging disease. Centre for Infection Medicine (ZIM), University of Veterinary Medicine, Hannover, Foundation, 9 July 2012, Hannover, Germany.

15. Elbers ARW, et al. Seroprevalence of Schmallenberg virus antibodies among dairy cattle, the Netherlands, winter 2011-2012. Emerging Infectious Diseases 2012; 18: $1065-1071$.

16. Kettler DS. Biology and bionomics of bloodsucking Ceratopogonids. Annual Review of Entomology 1977; 22: $33-51$.

17. Kiel E, et al. Monitoring of Culicoides at 20 locations in northwest Germany. Parasitology Research 2009; 105: $351-357$.

18. Ander M, Meiswinkel R, Chirico J. Seasonal dynamics of biting midges (Diptera: Ceratopogonidae: Culicoides), the potential vectors of bluetoungue virus, in Sweden. Veterinary Parasitology 2012; 184: 59-67.

19. Gonzáles M, et al. A survey of Culicoides developmental sites on a farm in northern Spain, with a brief review 
of immature habitats of European species. Veterinary Parasitology 2013; 191: 81-93.

20. Mehlhorn H, et al. Entomological survey on vectors of Bluetongue virus in North Rhine-Westfalia (Germany) during 2007 and 2008. Parasitology Research 2009; 105: 321-329.

21. Rüsse I, Grunert E. Normal gravidity: implantation and placentation of ruminants. In: Richter J, Götze R, eds. Tiergeburtshilfe. Berlin/Hamburg: Verlag Paul Parey, 1993, p. 45.

22. Rüsse I, Grunert E. Normal gravidity: uniparous state and multiparous state in small runinants. In: Richter J, Götze R, eds. Tiergeburtshilfe. Berlin/Hamburg: Verlag Paul Parey, 1993, pp. 31-35.

23. Schmahl G, Klimpel S, Walldorf V. Pilot study on deltamethrin treatment $\left(\right.$ Butox $^{\circledR} 7 \cdot 5$, Versatrine ${ }^{\circledR}$ ) of cattle and sheep against midges (Culicoides species, Ceratopogonidae). Parasitology Research 2009; 104: 809-813.
24. Schmahl G, Mehlhorn H. Does rain reduce the efficacy of Butox 7.5 pour on (Deltamethrin) against biting midges (Culicoides specimens)? Parasitology Research 2009; 105: 1763-1765.

25. Venail AR, et al. Laboratory and field-based tests of deltamethrin insecticides against adult Culicoides biting midges. Journal of Medical Entomology 2011; 48: 351-357.

26. Griffioen K, et al. Culicoides species associated with sheep in the Netherlands and the effect of a permethrin insecticide. Veterinary Journal 2011; 190: 230-235.

27. Doherty WM, et al. Protection of cattle from Culicoides spp. in Australia by shelter and chemical treatment. Veterinaria Italiana 2004; 40: 320-323.

28. Baldet $\mathbf{T}$, et al. Indoor activity of Culicoides spp. in association with livestock in the bluetongue virus (BTV) affected region of Northern France during autumn 2006. Preventative Veterinary Medicine 2008; 87: 84-97. 